\title{
Study on microbial depolymerization processes of xenobiotic polymers with mathematical modelling and numerical simulation
}

\author{
Masaji Watanabe $^{1} \quad$ Fusako Kawai $^{2}$
}

(Received 9 January 2012; revised 24 May 2012)

\begin{abstract}
Microbial depolymerization processes of polyethylene glycol are studied using a model for general depolymerization processes. The model involves a degradation rate that is a product of a time factor and a molecular factor. An inverse problem is solved numerically to determine the time factor using weight distributions with respect to the molecular weight before and after cultivation of a microbial consortium. The time factor is the microbial population whose carbon source is the liberated monomers. An initial value problem is solved numerically to simulate the transition of the weight distribution and the microbial population.
\end{abstract}

http://journal.austms.org.au/ojs/index.php/ANZIAMJ/article/view/5107 gives this article, (c) Austral. Mathematical Soc. 2012. Published June 13, 2012. ISSN 1446-8735. (Print two pages per sheet of paper.) Copies of this article must not be made otherwise available on the internet; instead link directly to this URL for this article. 


\section{Contents}

1 Introduction

C204

2 Modeling exogenous type depolymerization processes

C205

3 Removal of time dependence from the degradation rate

C208

4 Memoryless behaviour in liberation of monomers

C209

5 Computational results for a depolymerization process

C210

6 Discussion

$\mathrm{C} 212$

References

C214

\section{Introduction}

Microbial depolymerization processes are classified into exogenous type processes and endogenous type processes. In an exogenous depolymerization process, molecules reduce in size by liberation of monomers from their terminals. Polymers subject to exogenous depolymerization processes include polyethylene (PE). The PE biodegradation involves two essential factors: the gradual weight loss of large molecules due to $\beta$-oxidation; and the direct consumption or absorption of small molecules by cells. A mathematical model based on those factors was proposed to analyse PE biodegradation [5, 6, 20].

Polyethylene glycol (PEG) is another polymer subject to exogenous depolymerization processes. PEG is one of the polyethers whose chemical structures are expressed by $\mathrm{HO}(\mathrm{R}-\mathrm{O})_{\mathfrak{n}} \mathrm{H}$, for example,

- PEG, $\mathrm{R}=\mathrm{CH}_{2} \mathrm{CH}_{2}$,

- $\mathrm{PPG}, \mathrm{R}=\mathrm{CH}_{3} \mathrm{CHCH}_{2}$, 
- ptmg, $\mathrm{R}=\left(\mathrm{CH}_{2}\right)_{4}$ [2].

PEG is metabolized by liberating $\mathrm{C}_{2}$ compounds [3, 4]. The mathematical techniques developed for PE biodegradation were extended to cover studies of the exogenous depolymerization processes of PEG [14]. Inverse problems were solved numerically to determine molecular factors of degradation rates based on the weight distribution of PEG with respect to molecular weight before and after cultivation of a microbial consortium $\mathrm{E} 1[14,16,17,18,19]$. Initial value problems were solved to simulate the transition of the weight distribution [14, $16,17,18,19]$. The time dependence of degradation rates was also studied in modelling and simulation of depolymerization processes of PEG [16, 17, 18]. A model originally developed for endogenous depolymerization processes is applied to the exogenous depolymerization processes of PEG [19].

In this study, PEG biodegradation is revisited. A mathematical model proposed in a previous study of the PE biodegradation [13] is applied to the PEG biodegradation. An inverse problem is solved numerically to determine a time factor of a degradation rate. An initial value problem is solved numerically to simulate transition of the weight distribution of PEG. Numerical techniques are illustrated and numerical results are presented.

\section{Modeling exogenous type depolymerization processes}

Molecules of a single polymer all have the same chemical structure, but their size may be different. Let $w(t, M)$ be the weight distribution of a polymer with respect to the molecular weight $M$ at time $t$. When a molecule with molecular weight $M$ is depolymerized in an exogenous type depolymerization process to become a molecule with molecular weight $\mathrm{K}$, the amount $\mathrm{M}-\mathrm{K}$ is metabolized. Let $p_{0}(t, K, M)$ be the time rate of increase in $w(t, K)$ due to the depolymerization of molecules with molecular weight $M, p_{1}(t, K, M)$ be the total amount metabolized in production of molecules with molecular weight $\mathrm{K}$ 
from molecules with molecular weight $M$, and $p(t, K, M)=p_{0}(t, K, M)+$ $p_{1}(t, K, M)$. Then $p(t, K, M)$ is the time rate of decrease in $w(t, M)$ to yield the increase $p_{0}(t, K, M)$ in $w(t, K)$. Let $C(A, B)$ be the class of all molecules whose molecular weights lie between $A$ and $B$. The weight decrease and the weight increase in $C(A, B)$ per unit time are

$$
\int_{A}^{B}\left(\int_{0}^{M} p(t, K, M) d K\right) d M \text { and } \int_{A}^{B}\left(\int_{M}^{\infty} p_{0}(t, M, K) d K\right) d M .
$$

The total weight in $C(A, B)$ present at time $t$ and its rate of change are

$$
\int_{A}^{B} w(t, M) d M \text { and } \frac{d}{d t} \int_{A}^{B} w(t, M) d M .
$$

The rate of change equals the difference between the quantities (1):

$$
\begin{aligned}
\frac{d}{d t} \int_{A}^{B} w(t, M) d M= & -\int_{A}^{B}\left(\int_{0}^{M} p(t, K, M) d K\right) d M \\
& +\int_{A}^{B}\left(\int_{M}^{\infty} p_{0}(t, M, K) d K\right) d M,
\end{aligned}
$$

where $A$ and $B$ are constants. The equation

$$
\frac{d}{d t} \int_{A}^{B} w(t, M) d M=\int_{A}^{B} \frac{\partial}{\partial t} w(t, M) d M
$$

holds for a function with continuous partial derivatives, and (2) becomes

$$
\int_{A}^{B}\left(\frac{\partial}{\partial t} w(t, M)+\int_{0}^{M} p(t, K, M) d K-\int_{M}^{\infty} p_{0}(t, M, K) d K\right) d M=0 .
$$

This equation holds for an arbitrary interval $[A, B]$, and the weight distribution $w=w(t, M)$ is a solution of the equation [13, 15, 21, 22].

$$
\frac{\partial w}{\partial t}=-\int_{0}^{M} p(t, K, M) d K+\int_{M}^{\infty} p_{0}(t, M, K) d K
$$


In the weight transition from $w(t, M)$ to $w(t, K)$ in an exogenous type depolymerization process, molecules with molecular weight $M$ becomes molecules with molecular weight $\mathrm{K}$, and the amount $\mathrm{M}-\mathrm{K}$ is consumed, while the number of degraded molecules is preserved. The number of degraded molecules is proportional to $(1 / M) p(t, K, M)$, and the increase in the $w(t, K)$ is $(K / M) p(t, K, M)$. Note that this amount equals $p_{0}(t, K, M)$ and substituting $(M / K) p(t, M, K)$ for $p_{0}(t, M, K)$, Equation (3) becomes

$$
\frac{\partial w}{\partial t}=-\int_{0}^{M} p(t, K, M) d K+\int_{M}^{\infty} \frac{M}{K} p(t, M, K) d K .
$$

Let $\gamma(t, M)$ be the loss of amount from $w(t, M)$ per unit time and per unit weight. The loss from $w(t, M)$ per unit time is $\gamma(t, M) w(t, M)$, and it is expressed in terms of the integral of $p(t, K, M)$,

$$
\gamma(t, M) w(t, M)=\int_{0}^{M} p(t, K, M) d K
$$

For $K \in[0, M]$, let $q(K, M)$ be the decrease in $w(t, M)$ per unit weight in the transition from $w(t, M)$ to $w(t, K)$. Then

$$
p(t, K, M)=\gamma(t, M) q(K, M) w(t, M)
$$

holds. From Equations (4), (5) and (6), the equation

$$
\frac{\partial w}{\partial t}=-\gamma(t, M) w+\int_{M}^{\infty} \frac{M}{K} \gamma(t, K) q(M, K) w(t, K) d K
$$

is obtained. Given an initial weight distribution $\mathbf{f}(\mathbf{M})$, Equation (7) and the initial condition

$$
w(0, M)=f(M)
$$

form an initial value problem, provided the degradation rate $\gamma(t, M)$ is given. Given an additional weight distribution $g(M)$ at $t=T(T>0)$, Equation (7), the condition (8), and the condition

$$
w(0, M)=g(M)
$$


form an inverse problem to determine the degradation rate $\gamma(t, M)$, for which the solution of the initial value problem (7) and (8) also satisfies the condition (9).

\section{Removal of time dependence from the degradation rate}

Time factors of degradability include temperature, dissolved oxygen, and microbial population. These factors act evenly on molecules regardless of sizes. The degradation rate $\gamma(t, M)$ is a product of a function of $t, \sigma(t)$, and a function of $M, \lambda(M)$. Equation (7) becomes

$$
\frac{\partial w}{\partial t}=-\sigma(t) \lambda(M) w+\sigma(t) \int_{M}^{\infty} \frac{M}{K} \lambda(M) q(M, K) w(t, K) d K .
$$

Define a change of variables by

$$
\tau=\int_{0}^{t} \sigma(s) d s \quad \text { and } \quad W(\tau, M)=w(t, M) .
$$

Then Equation (10) becomes

$$
\frac{\partial W}{\partial \tau}=-\lambda(M) W+\int_{M}^{\infty} \frac{M}{K} \lambda(K) q(M, K) W(\tau, K) d K .
$$

Given the molecular factor of the degradation rate $\lambda(M)$, Equation (11) and the initial condition

$$
W(0, M)=f(M)
$$

form an initial value problem. Equation (11), the initial condition (12) and the condition

$$
W(\mathcal{T}, M)=g(M)
$$

form an inverse problem to determine the molecular factor of the degradation rate $\lambda(M)$ for which the solution of the initial value problem (11) and (12) also 
satisfies the condition (13). The initial value problem (7) and (8) correspond to the initial value problem (11) and (12). The inverse problem (7), and (9) correspond to the inverse problem (11), (12) and (13), provided

$$
\mathcal{T}=\int_{0}^{T} \sigma(s) d s
$$

\section{Memoryless behaviour in liberation of monomers and exponential consumption of carbon source}

The liberation of monomers in an exogenous depolymerization process is memoryless; that is, the amount truncated from a molecule does not depend on the previous truncation. The memoryless behaviour leads to the exponential distribution $s(J)=\rho e^{-\rho J}$, [1] where $J$ is the amount truncated from a molecule in an exogenous depolymerization process. In an exogenous depolymerization process, an amount $\mathrm{M}-\mathrm{K}$ is liberated from a molecule with molecular weight $M$ to yield a molecule with molecular weight $K$. Then $q(K, M)=$ $s(M-K)=\rho e^{-\rho(M-K)}$, and Equation (11) becomes

$$
\frac{\partial W}{\partial \tau}=-\lambda(M) W+c(M) \int_{M}^{\infty} \lambda(K) d(K) W(\tau, K) d K,
$$

where

$$
c(M)=M e^{\rho M}, \quad d(K)=\frac{1}{K} e^{-\rho K} .
$$

The parameter $\rho$ is often called the intensity of the exponential distribution. In an exogenous type depolymerization process, molecules reduce in size through successive liberation of monomers. Let $L$ be the molecular weight of a monomer liberated from a molecule in one cycle of a depolymerization process. For the $\beta$-oxidation of $\mathrm{PE}, \mathrm{L}=28\left(\mathrm{CH}_{2} \mathrm{CH}_{2}\right)$, and for the PEG biodegradation, 
$\mathrm{L}=44\left(\mathrm{CH}_{2} \mathrm{CH}_{2} \mathrm{O}\right)$. Let $\mathrm{T}_{\mathrm{J}}$ be the time it takes for a molecule to reduce in size by the molecular weight $\mathrm{J}$. Since the liberation of monomer units occurs successively, $T_{2 L}=2 T_{L}$. The probability for loss of two monomer units in unit of time is one half of the probability for loss of one monomer unit, and $q(M-L, M)=2 q(M-2 L, M)$ or $\rho e^{-\rho L}=2 \rho e^{-2 \rho L}$, which leads to

$$
\rho=\frac{1}{\mathrm{~L}} \log 2 .
$$

\section{Computational results for a depolymerization process of polyethylene glycol}

Numerical techniques have been developed for the inverse problem to determine the degradation rate $\lambda(M)$ for which the solution of the initial value problem (14) and (12) also satisfies the condition (13) [19]. Figure 1(a) shows the transition of the weight distribution of PEG for seven days. Weight distributions before and after cultivation of the microbial consortium E1 for three days were set as the initial and final conditions, and the inverse problem was solved numerically. Figure 1(b) shows a numerical result for the molecular factor $\lambda(M)$.

Once the degradation rate is found, transition of the weight distribution is simulated by solving the initial value problem (14) and (12). Numerical techniques to solve the initial value problem have been developed in previous studies [15, 19, 21, 22]. Figure 2 shows numerical results. A time factor proposed previously [17] was used to simulate the transition of the weight distribution with the initial value problem (11) and (12). Numerical results are shown in Figure 3. 


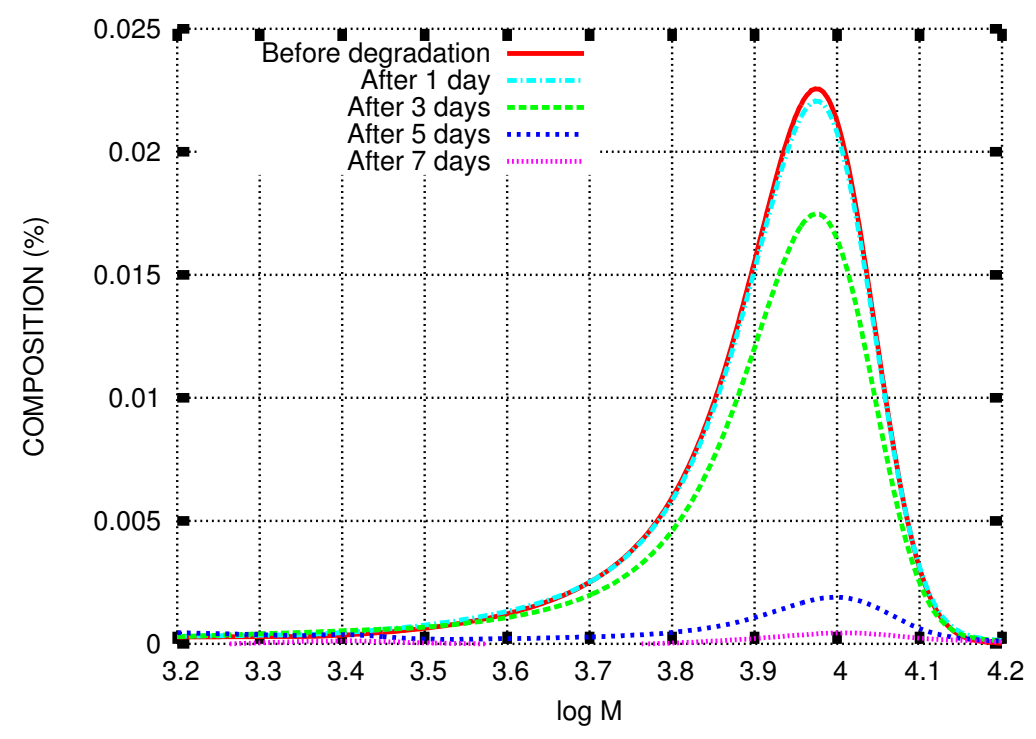

(a)

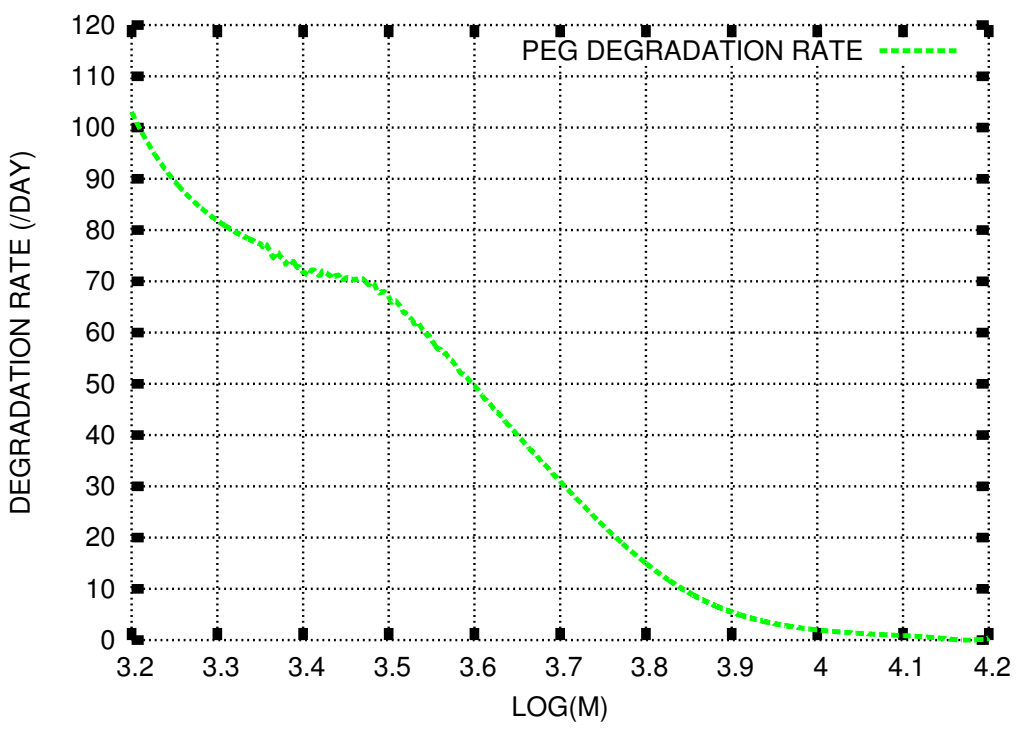

Figure 1: (a) Weight distribution of PEG before and after cultivation of microbial consortium E1 for seven days [18]. (b) Degradation rate based on the weight distributions before and after cultivation of the microbial consortium E1 on PEG for three days. 


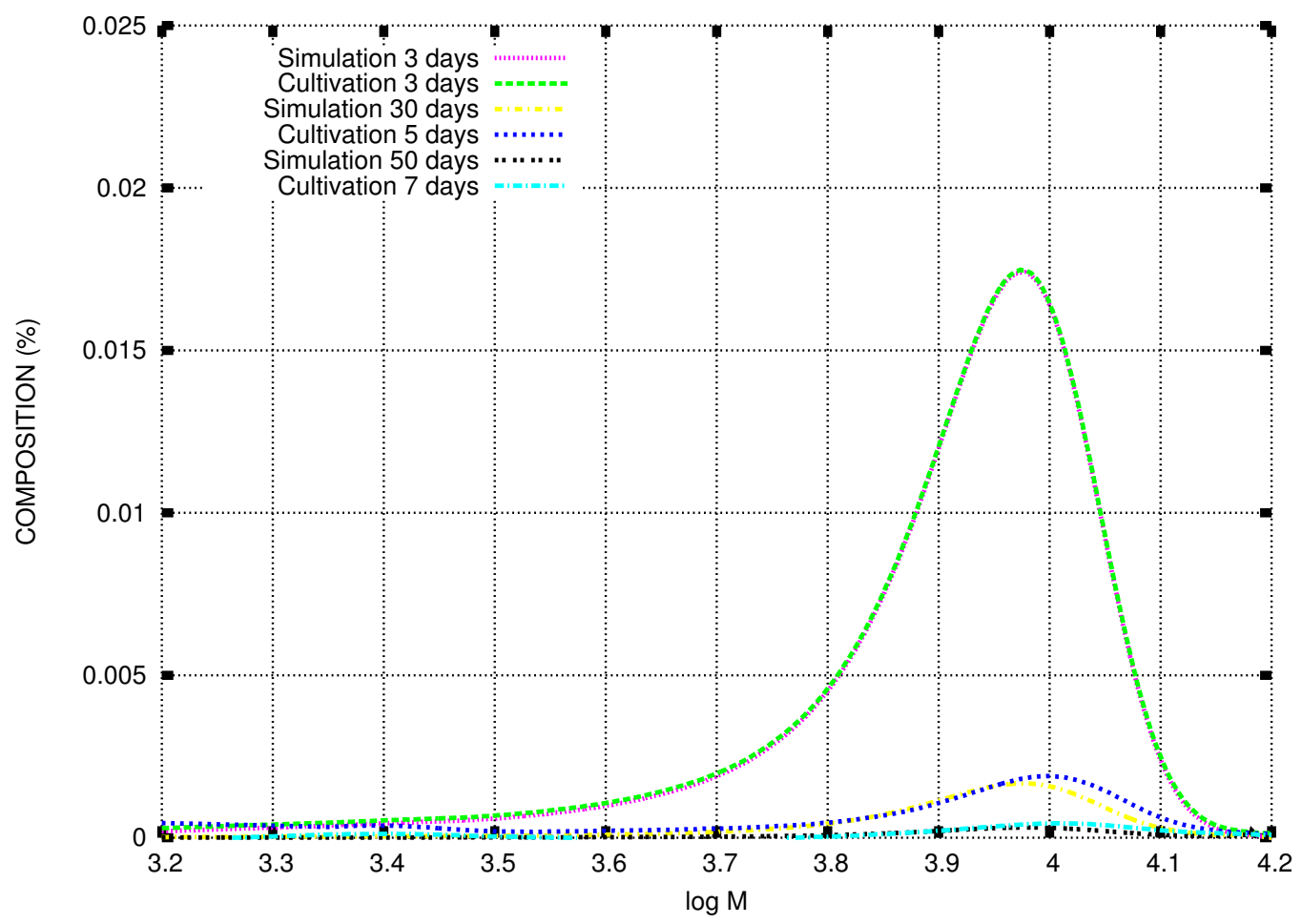

Figure 2: Experimental results and numerical results for transition of the weight distribution from $\tau=0$ to $\tau=50$.

\section{Discussion}

The molecular factor of the degradation rate $\lambda(M)$ shown in Figure $1(b)$ is the average degradation rate over the cultivation period for three days. Figure 2 shows that $t=3, t=5$, and $t=7$ correspond to $\tau=3, \tau=30$, and $\tau=50$, as we showed in previous studies with an exogenous depolymerization model $[18,19]$. The only time factor to obtain the experimental result was the microbial population. The numerical result indicates that the microbial population reached a peak after three days and that it started diminishing as 


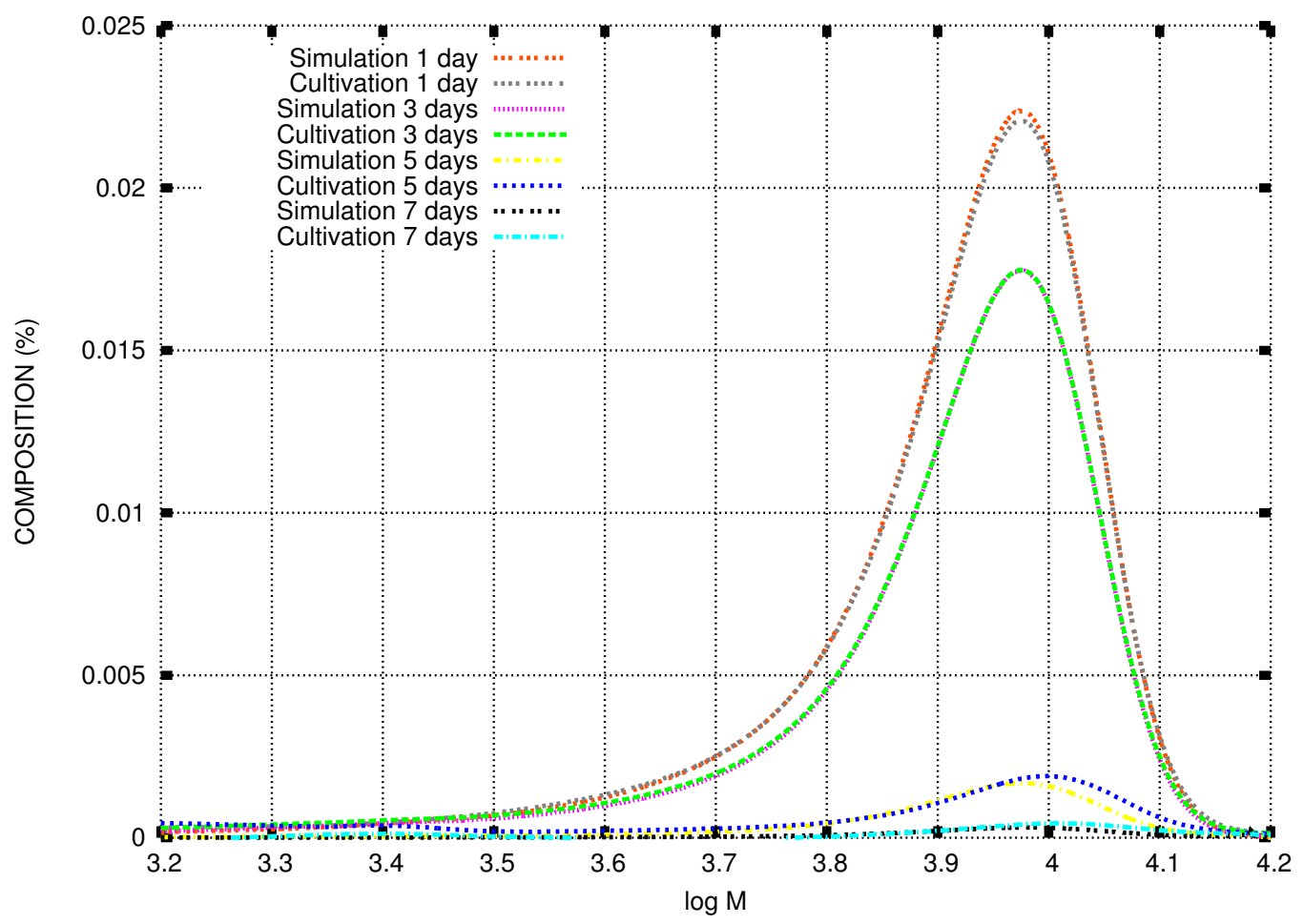

Figure 3: Experimental results and numerical results for transition of the weight distribution from $t=0$ to $t=7$.

the carbon source was exhausted.

The mathematical model originally developed for endogenous depolymerization processes is adapted to exogenous depolymerization processes of PE and PEG. Some others proposed models similar to the depolymerization model described in this study. They include governing equations for particle size distribution in simultaneous binary fragmentation and aggregation reactions or a population balance equation [7, 10, 11], equations for mass balance for polymers subject to random chain scission, repolymerization reactions, chain-end scission and related topics [8,9], and an integro-partial differential 
equation of a first order, bond breaking, process for random scission and a first order, recombination process [12].

The model based on the exponential distribution of degraded molecules is applied to PE biodegradation in a previous study. In this study, the model is applied to PEG biodegradation. Numerical results show that the model is widely applicable not only to endogenous depolymerization processes, but also to exogenous depolymerization processes of PEG biodegradation.

Acknowledgements: The authors thank Ms Y. Shimizu for her technical support. This work was supported by JSPS KAKENHI 20540118.

\section{References}

[1] Manfred Denker and Wojbor A. Woyczyński. Introductory Statistics and Random Phenomena, Uncertainty, Complexity and Chaotic Behavior in Engineering and Science, with Mathematica Uncertain Virtual Worlds by Bernard Ycart. Birkhäuser, Boston, 1998. C209

[2] F. Kawai. Biodegradability and chemical structure of polyethers. Kobunshi Ronbunshu, 50(10):775-780, 1993. In Japanese. C205

[3] F. Kawai. Breakdown of plastics and polymers by microorganisms. Advances in Biochemical Engineering/Biotechnology, 52:151-194, 1995. C205

[4] F. Kawai. Microbial degradation of polyethers. Applied Microbiology and Biotechnology, 58:30-38, 2002. doi:10.1007/s00253-001-0850-2 C205

[5] F. Kawai, M. Watanabe, M. Shibata, S. Yokoyama, and Y. Sudate. Experimental analysis and numerical simulation for biodegradability of polyethylene. Polymer Degradation and Stability, 76:129-135, 2002. doi:10.1016/S0141-3910(02)00006-X C204 
[6] F. Kawai, M. Watanabe, M. Shibata, S. Yokoyama, Y. Sudate, and S. Hayashi. Comparative study on biodegradability of polyethylene wax by bacteria and fungi. Polymer Degradation and Stability, 86:105-114, 2004. doi:10.1016/j.polymdegradstab.2004.03.015 C204

[7] G. Madras and B. J. McCoy. Numerical and similarity solutions for reversible population balance equations with size-dependent rates. Journal of Colloid Interface Science, 246:356-365, 2002. C213

[8] B. J. McCoy. Distribution kinetics for temperature-programmed pyrolysis. Ind. Eng. Chem. Res, 38:4531-4537, 1999. C213

[9] B. J. McCoy. Polymer thermogravimetric analysis: effects of chain-end and reversible random scission. Chemical Engineering Science, 56:1525-1529, 2001. C213

[10] B. J. McCoy and G. Madras. Evolution of similarity solutions for fragmentation and aggregation. Journal of Colloid Interface Science, 201:200-209, 1998. C213

[11] B. J. McCoy and G. Madras. Discrete and continuous models for polymerization and depolymerization. Chemical Engineering Science, 56:2831-2836, 2001. C213

[12] J. E. J. Staggs. A continuous model for vapourisation of linear polymers by random scission and recombination. Fire Safety Journal, 40:610-627, 2005. doi:10.1016/j.firesaf.2005.05.004 C214

[13] M. Watanabe and F. Kawai. Modeling biodegradation of polyethylene with memoryless behavior in metabolic consumption. Submitted. C205, C206

[14] M. Watanabe and F. Kawai. Numerical simulation of microbial depolymerization process of exogenous type. In Rob May and A. J. Roberts, editors, Proc. of 12th Computational Techniques and Applications Conference, CTAC-2004, Melbourne, Australia in September 2004, volume 46(E) of ANZIAM J., pages C1188-C1204, 
2005. http://journal.austms.org.au/ojs/index.php/ANZIAMJ/ article/view/1014 C205

[15] M. Watanabe and F. Kawai. Mathematical modelling and computational analysis for enzymatic degradation of xenobiotic polymers. Applied Mathematical Modelling, 30:1497-1514, 2006. doi:10.1016/j.apm.2005.12.011 C206, C210

[16] M. Watanabe and F. Kawai. Mathematical study of the biodegradation of xenobiotic polymers with experimental data introduced into analysis. In Andrew Stacey, Bill Blyth, John Shepherd, and A. J. Roberts, editors, Proceedings of the 7th Biennial Engineering Mathematics and Applications Conference, EMAC-2005, Melbourne, volume 47 of ANZIAM J., pages C665-C681, 2007. http://journal.austms.org. au/ojs/index.php/ANZIAMJ/article/view/1069 C205

[17] M. Watanabe and F. Kawai. Mathematical analysis of microbial depolymerization processes of xenobiotic polymers. In Geoffry N. Mercer and A. J. Roberts, editors, Proceedings of the 14th Biennial Computational Techniques and Application Conference, CTAC2008, volume 50 of ANZIAM J., pages C930-C946, 2009. http://journal. austms.org.au/ojs/index.php/ANZIAMJ/article/view/1465 C205, C210

[18] M. Watanabe and F. Kawai. Effects of microbial population in degradation process of xenobiotic polymers. In P. Howlett, M. Nelson, and A. J. Roberts, editors, Proceedings of the 9th Biennial Engineering Mathematics and Applications Conference, EMAC-2009, volume 51 of ANZIAM J., pages C682-C696, 2010. http://journal.austms.org. $\mathrm{au} /$ ojs/index.php/ANZIAMJ/article/view/2433 C205, C211, C212

[19] M. Watanabe and F. Kawai. Study on biodegradation of xenobiotic polymers with change of microbial population. In W. McLean and A. J. Roberts, editors, Proceedings of the 15th Biennial Computational Techniques and Applications Conference, CTAC-2010, volume 52 of 
ANZIAM J., pages C410-C429, 2011. http://journal .austms.org . au/ojs/index.php/ANZIAMJ/article/view/3965 C205, C210, C212

[20] M. Watanabe, F. Kawai, M. Shibata, S. Yokoyama, and Y. Sudate. Computational method for analysis of polyethylene biodegradation. Journal of Computational and Applied Mathematics, 161(1):133-144, December 2003. doi:10.1016/S0377-0427(03)0051-X C204

[21] Masaji Watanabe and Fusako Kawai. Numerical simulation for enzymatic degradation of poly(vinyl alcohol). Polymer Degradation and Stability, 81:393-399, 2003. doi:10.1016/S0141-3910(03)00122-8 C206, C210

[22] Masaji Watanabe, Fusako Kawai, Sadao Tsuboi, Shogo Nakatsu, and Hitomi Ohara. Study on enzymatic hydrolysis of polylactic acid by endogenous depolymerization model. Macromolecular Theory and Simulations, 16:619-626, 2007. doi:10.1002/mats.200700015 C206, C210

\section{Author addresses}

1. Masaji Watanabe, Graduate School of Environmental Science, Okayama University, Okayama, JAPAN. mailto:watanabe@ems.okayama-u.ac.jp

2. Fusako Kawai, Center for Nanomaterials and Devices, Kyoto Institute of Technology JAPAN. 